\title{
Achieving Fair Spectrum Allocation and Reduced Spectrum Handoff in Wireless Sensor Networks: Modeling via Biobjective Optimization
}

\author{
Sang-Seon Byun, ${ }^{1}$ Kimmo Kansanen, ${ }^{2}$ Ilangko Balasingham, ${ }^{3}$ and Joon-Min Gil ${ }^{4}$ \\ ${ }^{1}$ Department of Computer Engineering, Catholic University of Pusan, Busan, Republic of Korea \\ ${ }^{2}$ Department of Electronics and Telecommunications, Norwegian University of Science and Technology, Trondheim, Norway \\ ${ }^{3}$ Rikshospitalet, Oslo University Hospital, Oslo, Norway \\ ${ }^{4}$ School of Information Technology Engineering, Catholic University of Daegu, Gyeongsan, Gyeongbuk, Republic of Korea
}

Correspondence should be addressed to Joon-Min Gil; jmgil@cu.ac.kr

Received 26 June 2014; Accepted 12 August 2014; Published 3 September 2014

Academic Editor: James J. Park

Copyright (C) 2014 Sang-Seon Byun et al. This is an open access article distributed under the Creative Commons Attribution License, which permits unrestricted use, distribution, and reproduction in any medium, provided the original work is properly cited.

\begin{abstract}
This paper considers the problem of centralized spectrum allocations in wireless sensor networks towards the following goals: (1) maximizing fairness, (2) reflecting the priority among sensor data, and (3) avoiding unnecessary spectrum handoff. We cast this problem into a multiobjective mixed integer nonconvex nonlinear programming that is definitely difficult to solve at least globally without any aid of conversion or approximation. To tackle this intractability, we first convexify the original problem using arithmetic-geometric mean approximation and logarithmic change of the decision variables and then deploy weighted Chebyshev norm-based scalarization method in order to collapse the multiobjective problem into a single objective one. Finally, we apply simple rounding method in order to obtain approximate integer solutions. The results obtained from the numerical experiments show that, by adjusting the weight on each objective function, the proposed algorithm allocates spectrum bands fairly with well observing each sensor's priority and reduced spectrum handoffs.
\end{abstract}

\section{Introduction}

The demand of allocating and using the radio frequency spectra is rapidly growing due to increasing number of wireless and mobile communication applications, where the industry has reached the limits of current static spectrum allocation. However, actual measurements illustrate that the scarcity is not a result of heavy usage of the spectrum. It is merely due to the inefficiency of the static spectrum allocation pursued by regulators [1].

Dynamic spectrum allocation may resolve this paradox by opening assigned, but sparsely used, spectrum resources to secondary users [2-4]. It exploits underutilized spectrum resources along time or frequency dimension and provides efficient and intensive dynamic spectrum access through the detection of spectrum opportunity and adaptive modulation.

Such dynamic spectrum access schemes can be considered in wireless sensor networks (WSNs) as well. Typical
WSNs are composed of resource-constrained sensors responsible for monitoring physical phenomena and reporting to sink points. One of the primary objectives of WSNs is to transmit monitored results timely and concurrently, without using large amount of network resources. The dynamic spectrum access becomes very vital to achieve such timely and concurrent transmissions in WSNs; for instance, in a WSN for real time surveillance system $[5,6]$ or real time machine-to-machine communications [7], the transmissions of video or image data captured by the sensors require high bandwidth and multiple spectra [8]. Subsequently, the following major principles can be made.

1.1. Fair Allocation of Idle Spectrum Bands. If too many sensors attempt to transmit their data simultaneously, current idle spectrum resources may be insufficient to support all transmissions. In this situation, scarce spectrum resources 
should be allocated as fairly as possible; in addition, it is necessary to prioritize the transmissions. This can be achieved by maximizing proportional fairness with demanding weights [9]. The main difference between max-min fairness and proportional fairness is that the latter is in a sense less fair in terms of the demanding volume assignment but more effective in terms of the total utilization achieved by all participants [10].

1.2. Avoiding Unnecessary Spectrum Handoff. The other primary objective of WSNs is long time functionality. However, dynamic spectrum access leads to supplemental energy consumption at each sensor due to spectrum handoff. Spectrum handoff occurs when (1) primary user is detected or (2) current spectrum condition becomes worse; sensors move to the "best matched" available spectrum band. It has been measured that not only decoding and channel estimation but also frequency or timing synchronization consumes a certain amount of power [11]. It means that the more frequent spectrum handoffs lead to the more power consumption. Moreover, due to the latency caused by spectrum sensing, decision, and handoff procedures, quality degradation is inevitable during spectrum handoff [12]. Hence unnecessary spectrum handoff should be eliminated $[13,14]$.

1.3. Centralized Spectrum Allocation. The problem of dynamic spectrum allocation in cellular network has been studied widely. Various centralized or distributed algorithms are summarized and compared in [15]. In centralized scheme, a centralized authority (e.g., base station or dedicated coordinator) detects and identifies spectrum opportunities and allocates the identified spectra to secondary users in accordance with a predefined policy. All these procedures may be done separately by different entities, that is, detector, identifier, and allocator. Undoubtedly there is an overhead of message exchange since the centralized authority (or allocator) should propagate the results of spectrum allocation into sensor swarm whenever it receives transmission requests. In addition, if sensors are distributed in a wide area, more detectors may be needed.

In distributed scheme, each secondary user competes with one another to access available spectrum resources. Thus each user should have an ability to detect the spectral opportunities and determine an optimal strategy to maximize its benefits (e.g., number of spectrum bands). If the distributed scheme is deployed to WSN, all sensors should have a fully operating cognitive radio. However it is not feasible to implement full features of cognitive radio in such energylimited sensor nodes. Thus, in a moderate size of WSN, where sensors are not distributed widely, for example, a healthcare system in an intensive care unit, the centralized scheme is preferred to the distributed one.

In this paper, we consider the problem of the centralized spectrum allocations in a WSN of moderate size; that is, all sensors are located within a cell or segment boundary. We assume that sensors, which are going to transmit data immediately or in near future, request spectrum resources to their dedicated coordinator. Then the coordinator allocates each sensor spectrum resources: (1) as fairly as possible, (2) reflecting the priority among sensors, and (3) avoiding unnecessary spectrum handoff. We formulate this problem into a multiobjective, more clearly, bicriteria and biobjective, mixed integer nonlinear nonconvex programming that is, however, known as intractable without any aid of modification or approximation.

Our approach to tackle this problem is summarized as follows. First, we convert the original formulation into a quasiequivalent form that is convex through arithmeticgeometric mean approximation and logarithmic change of decision variables. Then we relax the integer constraint, so-called NLP relaxation, and collapse the multiobjective optimization problem (MOP) into a single objective one using scalarization based on weighted Chebyshev norm (also called supremum or infinity norm [16]) problem by which we can maintain the convexity and achieve NLP relaxed solution which satisfies weak Pareto optimality. Finally, we perform simple rounding algorithm on the NLP relaxed solutions in a sequence of steps in order to obtain approximated integer solutions. A noticeable advantage of the proposed approach is that it enables finding fairly good approximated integer solutions within reasonable computation time. It is already proved that approximate solution obtained by the series of arithmetic-geometric mean approximations converges to a point satisfying the Karush-Kuhn-Tucker conditions of the original problem [17]. We estimate the integer solution of rounding method by comparing it with NLP relaxed solution that gives crude upper bounds of the original problem.

The rest of this paper is organized as follows. In Section 2, we present the related work and summarize our main challenges. In Section 3, the problem formulation is given. In Section 4, we describe the procedure of tackling the intractability: convexification, scalarization, and rounding. In Section 5, the results of numerical experiments are described. Finally we conclude this paper in Section 6.

\section{Related Work}

In [18], the authors consider the deployment of cognitive radio to WSNs and suggest that, by adjusting the constellation size, different data rate can be achieved which will directly influence the power consumption of each node and in turn will affect the lifetime of the whole sensor network. They deploy a distributed spectrum allocation strategy with the assumption that the full functions of cognitive radio, that is, detection, identification, and exploitation of spectrum opportunities, are installed at each sensor node. In [19], the authors apply dynamic spectrum access in the time domain by exploiting white spaces between bursty transmissions of multiaccess communication channels. They mention that if sensors communicate sporadically and at a low rate, it appears reasonable to assume that such systems could efficiently reuse the remaining white spaces.

There are many research literatures that have investigated the centralized spectrum allocation. We list some of them below. 
In [20], a scheme to achieve proportional fair rate allocation in multiuser OFDMA system is proposed. The authors deal with fully exclusive subcarrier allocation without any consideration of interference. They apply heuristic algorithm of relatively high complexity.

In [21,22], the dynamic spectrum allocation among base stations is considered. In [21], the authors apply genetic algorithm in order to allocate spectra while maximizing the spectral efficiency and satisfying each base station's service rate. However, the genetic algorithm is not appropriate for a multiobjective optimization with multiple constraints since if we consider only a population that satisfies all constraints during the entire procedure of the genetic algorithm, the solutions tend to be stagnated at the local optimal [23]. If we use the algorithm without concerning any of the constraints, few solutions subject to all the constraints can be obtained. In [22], the authors propose dynamic spectrum planning that reflects the volume of supported demand at each base station based on its distribution statistics and local interference condition, and they also address a long-term stability and the proportional fairness.

In $[24,25]$, downlink channel (or subcarrier) allocation problems are considered, and low complex heuristic-based algorithms are proposed in order to solve the problem in reasonable time. The main objectives are to minimize the required transmit power while satisfying the rate requirement and data error rate constraint of each user [24] and maximize the total number of active subcarriers that can be supported to unlicensed users [25], respectively.

In [26], the authors propose an intuitional heuristic algorithm for proportional fair rate allocation. They express the problem using nonlinear convex programming and estimate the heuristic algorithm by comparing its results with the optimal solution.

In [27], the authors model the joint power control and rate allocation problem in a multiuser CDMA system as geometric programming, which can be converted into a convex form easily. They have also considered the proportional fair rate allocations.

In [28], the authors prove that the region of feasible signal-to-inference ratio (SIR) is strictly convex if it has logarithmic scale. As a result, they show that the problem of finding a power vector that maximizes a weighted sum of logarithmic SIR is strictly convex, which coincides with the power control problem of achieving maximal weighted proportional fair SIR among users.

In [29], the authors have considered a game theory based bandwidth allocation mechanism in WiMax environments.

Comparing to the abovementioned related work, our main contributions can be highlighted as follows. We consider the issue of spectrum handoff, which means the problem is formulated as having discrete variables. A separate objective function has been established for the spectrum handoff instead of expressing it as a constraint in order to get several active solutions. (If we include the spectrum handoff in the constraint set, the number of spectrum handoffs will be bounded to a parameter that cannot be tuned during the problem solving in order to achieve reduced spectrum handoffs. Therefore, if the decision maker expects the active improvements in two or more objectives simultaneously by only one-shot problem solving, it is inevitable to model the problem into an MOP [16].) Accordingly, the problem is formulated as a multiobjective mixed integer nonlinear programming (MO-MINLP). Furthermore, convexification, scalarization, and rounding method have been employed to make the problem more tractable.

\section{Problem Formulation}

We formulate our centralized spectrum allocation problem as an MO-MINLP. Prior to describing the formulation, the following assumptions are made.

(1) Available spectrum resources are expressed as a number of spectrum units, and the bandwidth of each spectrum unit is fixed, for example, subcarrier in OFDM or subband in multiband ultrawide band (UWB).

(2) Each sensor can transmit over noncontiguous frequency bands concurrently $[20,24,30]$.

(3) Similar to $[26,27]$, each receiver sensor declares its desired signal-to-interference noise ratio (SINR). The SINR value reflects a specific required level of bit error rate (BER) since there is an explicit relationship between BER and SINR according to modulation schemes.

(4) All sensors can access entire idle spectrum resources. It means that sensors are distributed within the cell or segment boundary where primary nodes are associated.

(5) Similar to general centralized methods, the detection of spectrum opportunities, identification, and exploitation are always correct, and a dedicated coordinator does all these processes. Furthermore, the dedicated coordinator also lies within the same cell or segment boundary with the sensors.

(6) The process of the spectrum allocation is performed in every start of predefined epoch, where the coordinator detects idle spectrum units and the sensors that requested spectrum resources. The epoch may also start whenever the coordinator receives demands of spectrum units or periodically. Moreover, there is no change in the channel condition within each epoch.

(7) We consider direct communications among only sensors as shown in [31].

(8) Actually, a spectrum handoff occurs at a receiver as well as a transmitter. However, it is impossible to avoid the handoffs in both transmitter and receiver if both were not coupled in the last epoch and currently synchronized with different spectrum units. Hence, in this paper, we consider the spectrum handoff at only a transmitter. 
Based on the above assumptions, the MO-MINLP can be described as follows.

\section{Parameters}

(i) $V$ : set of sensors that request spectrum units for their transmissions.

(ii) S: set of idle spectrum units.

(iii) $L_{i s}$ (binary): it indicates that spectrum unit $s$ was used by sensor $i$ at its previous transmission phase. It means that, currently, sensor $i$ is synchronized with the spectrum unit $s$. We assume that the sets $S$ and $V$ vary in every transmission epoch. Thus we suppose that the current $L$ reflects the optimal solution of right previous epoch.

(iv) $w_{i}$ : weight given in accordance with the priority of sensor $i$. The higher priority a sensor has, the more spectrum units will be allocated. It means that the priority reflects the demand of spectrum units.

(v) $P_{i}^{\max }$ : maximal transmission power available at sensor $i$.

(vi) $\mathrm{INSR}^{\mathrm{max}}: 1 / \mathrm{SINR}^{\mathrm{min}}$, where $\mathrm{SINR}^{\mathrm{min}}$ is the minimal SINR that corresponds to strict QoS constraint.

(vii) $G_{i j}^{s}$ : channel gain between sensors $i$ and $j$ over spectrum unit $s$.

\section{Decision Variables}

(i) $x_{i s}$ (binary): it indicates that sensor $i$ occupies spectrum unit $s$.

(ii) $p_{i s}$ : transmission power for sensor $i$ in spectrum unit $s$.

(iii) INSR $_{i s}$ : reciprocal of SINR of sensor $i$ in spectrum unit $s$, defined as

$$
\mathrm{INSR}_{i s}=\frac{\sum_{j \in V \backslash\{i\}} x_{j s} p_{j s} G_{j k}^{s}+\sigma^{2}}{p_{i s} G_{i k}^{s}},
$$

where $\sigma^{2}$ is noise power.

Objectives. Consider the following:

$$
\begin{gathered}
\text { Maximize } f_{1}=\sum_{i \in V} w_{i} \ln \left(\sum_{s \in S} x_{i s}\right) \\
\text { Maximize } f_{2}=\sum_{s \in S} \sum_{i \in V} L_{i s} x_{i s}
\end{gathered}
$$

s.t.

$$
\begin{gathered}
c_{1}: x_{i s} \mathrm{INSR}_{i s} \leq \mathrm{INSR}_{i}^{\max } \quad \forall i \in V, s \in S, \\
c_{2}: \sum_{s \in S} x_{i s} p_{i s} \leq P_{i}^{\max } \quad \forall i \in V, \\
c_{3}: x_{i s} \in\{0,1\} \quad \forall i \in V, s \in S .
\end{gathered}
$$

According to the definition in [20], a resource allocation scheme $P$ is proportionally fair if and only if, for any other feasible allocation scheme $M$, we have

$$
P=\arg \max _{M} \sum_{i \in V} w_{i} \ln R_{i}^{(M)},
$$

where $w_{i}$ is the weight of user $i$ and $R_{i}^{(M)}$ is the average resource of user $i$ by an allocation scheme $M$. The weight reflects each user's priority to the proportional fair allocation as well (i.e., we give more resources to the user with higher priority) $[9,28]$.

Therefore, by maximizing $f_{1}$, we can achieve maximal proportional fairness in terms of the number of spectrum units allocated to each sensor and reflect the priority of each sensor simultaneously.

By maximizing $f_{2}$, we can let each sensor keep holding the spectrum units used in the previous transmission epoch. Constraint $c_{1}$ indicates that there is a corresponding SINR threshold that determines whether or not packet transmission is successful. That is, if $c_{1}$ is satisfied then the intended receiver can receive the packet correctly, and, otherwise, the packet is lost [32]. Constraint $c_{2}$ indicates that no sensor can use more transmission power than its maximal available transmission power.

Unlike the general fair "rate" allocation schemes [20, 26, 27], we consider fair "spectrum unit" allocations here due to the following reason: as shown in constraint $c_{2}$, each sensor is guaranteed to achieve SINR at least larger than SINR ${ }^{\text {min }}$. Since the SINR values directly correspond to the rates and if it is guaranteed that each sensor achieves the exact same SINR as SINR ${ }^{\mathrm{min}}$, the fair spectrum unit allocation will yield the "coarse-grained" fair rate allocation.

However, the problem is intractable due to its nonconvexity and discrete variables. For this reason, we manipulate the objective functions and apply a series of approximations.

Henceforth, we denote the single objective optimization problem with only the objective function $f_{1}$ and the constraints as $\Omega\left(f_{1}\right)$ and the one with only $f_{2}$ and the constraints as $\Omega\left(f_{2}\right)$, respectively. In addition, we denote the problem by both the objectives and the constraints as $\Omega\left(f_{1}, f_{2}\right)$.

\section{Tackling the Intractability}

We begin the convexification process with relaxing the integer constraint; that is, we drop (6).

\subsection{Convexification of $\Omega\left(f_{1}\right)$ and $\Omega\left(f_{2}\right)$}

4.1.1. Convexification of $\Omega\left(f_{1}\right)$. We convert the original function $f_{1}$ into an equivalent log-sum-exp function form that is proved to be convex $[17,27,33]$ by defining $\beta_{i s}=\ln \left(x_{i s}\right)$ and $\gamma_{i s}=\ln \left(p_{i s}\right)$ for all $i \in V$ and $s \in S$, where $-\infty \leq \beta_{i s} \leq 0$ and $-\infty \leq \gamma_{i s} \leq \ln \left(P_{i}^{\max }\right)$. Then $\Omega\left(f_{1}\right)$ is reformulated as follows.

Objective. Consider the following:

$$
\text { Maximize } f_{1}^{*}=\sum_{i \in V} w_{i} \ln \left(\sum_{s \in S} e^{\beta_{i s}}\right)
$$


Step 1. Generate initial feasible solution vectors $\beta$ and $\boldsymbol{\gamma}$.

Step 2. For all $i \in V$ and $s \in S$, evaluate $\alpha_{i s}$ with the initial vectors

$\beta$ and $\gamma$ using (11).

Step 3. Condense the objective function $f_{1}^{*}$ into $C v\left(f_{1}^{*}\right)$.

Step 4. Solve the resulting NLP.

Step 5. Terminate the $k$ th loop if $\left|f_{1}^{*}(k)-f_{1}^{*}(k-1)\right| \leq \omega$. Otherwise

go to Step 2 with solution vectors $\boldsymbol{\beta}$ and $\boldsymbol{\gamma}$ obtained at Step 4 .

Algorithm 1: Condensation algorithm.

s.t.

$$
\begin{aligned}
& C v\left(c_{1}\right) \\
& \quad: \ln \left(\sum_{j \in V \backslash\{i\}}\left(\begin{array}{c}
e^{\left(\beta_{i s}+\beta_{j s}+\gamma_{j s}-\gamma_{i s}\right)} G_{j k}^{s}\left(G_{i k}^{s}\right)^{-1}\left(\mathrm{INSR}_{i}^{\max }\right)^{-1} \\
+e^{\left(\beta_{i s}-\gamma_{i s}\right)} \sigma^{2}\left(G_{i k}^{s}\right)^{-1}\left(\mathrm{INSR}_{i}^{\max }\right)^{-1}
\end{array}\right)\right) \\
& \quad \leq 0
\end{aligned}
$$

$\forall i \in V, \quad s \in S$,

$$
C v\left(c_{2}\right): \ln \left(\sum_{s \in S} e^{\left(\beta_{i s}+\gamma_{i s}\right)}\left(P_{i}^{\max }\right)^{-1}\right) \leq 0 \quad \forall i \in V .
$$

Nonetheless the reformulated one is not a convex optimization [33]. Therefore, we deploy arithmeticgeometric mean approximation (in geometric programming, arithmetic-geometric mean approximation is used for condensing a posynomial function into a monomial function; therefore it is called also local monomial approximation [34]) $[17,34]$ as

$$
\sum_{s \in S} e^{\beta_{i s}} \geq \prod_{s \in S}\left(\frac{e^{\beta_{i s}}}{\alpha_{i s}}\right)^{\alpha_{i s}}
$$

and the inequality becomes an equality if

$$
\alpha_{i s}=\frac{e^{\beta_{i s}}}{\sum_{s \in S} e^{\beta_{i s}}}, \quad \forall i \in V, s \in S .
$$

Then the objective is condensed as

$$
\begin{aligned}
\operatorname{Maximize} C v\left(f_{1}^{*}\right) & =\sum_{i \in V} w_{i} \ln \left(\prod_{s \in S}\left(\frac{e^{\beta_{i s}}}{\alpha_{i s}}\right)^{\alpha_{i s}}\right) \\
& =\sum_{i \in V} w_{i} \sum_{s \in S} \alpha_{i s}\left(\beta_{i s}-\ln \alpha_{i s}\right),
\end{aligned}
$$

which becomes affine, and the optimization problem can be solved by condensation algorithm, Algorithm 1 [17].

As condensing the objective function $f_{1}^{*}$ into $C v\left(f_{1}^{*}\right)$ always yields underestimated solutions, each NLP in the condensation iteration loop tries to improve the accuracy of the approximation to a particular maximum in the original feasible region. In addition, the algorithm is convergent and always produces the approximate solution that satisfies the Karush-Kuhn-Tucker conditions of the original problem [17].
4.1.2. Convexification of $\Omega\left(f_{2}\right)$. As done in the convexification of $\Omega\left(f_{1}\right)$, we let $\beta_{i s}=\ln \left(x_{i s}\right)$ and $\gamma_{i s}=\ln \left(p_{i s}\right)$ for all $i \in V$ and $s \in S$, where $-\infty \leq \beta_{i s} \leq 0$ and $-\infty \leq \gamma_{i s} \leq \ln \left(P_{i}^{\max }\right)$. Then $f_{2}$ is converted into a convex form:

$$
\operatorname{Minimize} C v\left(f_{2}\right)=\sum_{s \in S} \sum_{i \in V} L_{i s} e^{-\beta_{i s}}
$$

The above formulation makes $\beta_{\text {is }}$ close to 0 , where $L_{i s}=1$ in order to minimize its value. Thus we can derive the same results as the ones obtained by maximizing $f_{2}$.

4.2. Scalarization of the Multiobjective Optimization Problem. For solving MOPs, one of the most widespread approaches is scalarization, where MOPs are replaced by suitable scalar optimization (i.e., single objective optimization) problems involving possibly some additional parameters and constraints. With the help of the scalar problem, not only can one optimal solution of the multiobjective optimization problem be found but also approximations of the whole solution set can be generated by a variation of the parameters. In this paper, we are interested in maintaining the convexity of the scalarized problem as well as guaranteeing weak Pareto optimality at least by minimizing the distance between ideal solutions (i.e., Pareto Frontier) and feasible objective region. To this end, we deploy a scalarization method based on weighted Chebyshev norm problem [16].

We consider an MOP with a vector of decision variables $\mathbf{x}$ and a vector of objective functions $\mathbf{f}(\mathbf{x})=\left[f_{1}(\mathbf{x}), \ldots, f_{r}(\mathbf{x})\right]^{T}$ as follows.

Objectives. Consider the following:

$$
\text { Minimize } f_{m}(\mathbf{x}), \quad m=1, \ldots, r
$$

s.t.

$$
h_{l}(\mathbf{x}) \leq 0, \quad l=1, \ldots, t
$$

To begin with, we normalize each $m$ th objective function $f_{m}$ to be given 0 as the minimum value and 1 as the maximum value as follows:

$$
f_{m n}(\mathbf{x})=\frac{f_{m}(\mathbf{x})-f_{m}\left(\mathbf{x}_{m}^{*}\right)}{f_{m w}(\mathbf{x})-f_{m}\left(\mathbf{x}_{m}^{*}\right)}
$$


where $f_{m w}=\max _{j=1, \ldots, r} f_{j}\left(\mathbf{x}_{j}^{*}\right)$ and $\mathbf{x}_{j}^{*}$ is the optimal design vector obtained when only $f_{j}$ is minimized. That is, the distance between its optimal solution and worst solution normalizes each objective function. Then the weighted Chebyshev norm problem is defined by additional variable $z$ as follows.

Objectives. Consider the following:

Minimize $z$

s.t.

$$
\begin{gathered}
\delta_{m} f_{m n}(\mathbf{x}) \leq z, \quad \forall m=1, \ldots, r, \\
h_{l}(\mathbf{x}) \leq 0, \quad l=1, \ldots, t,
\end{gathered}
$$

where $\delta_{m}>0$ is the weight of $f_{m}$ (notice that the $\delta_{m}$ expresses relative weight rather than absolute weight). By solving this scalarized problem, the maximal distance between the ideal solution $\left(f_{m}\left(\mathbf{x}_{m}^{*}\right)\right)$ and the feasible objective region $\left(f_{m}(\mathbf{x})\right)$ is minimized. (This scalarized problem corresponds to the problem that achieves the weighted fairness between objective values within min-max (i.e., supremum, and also called maxminimization) criterion. There is another scalarization approach that considers the proportional fairness between objective values [35]. But it fails to maintain the convexity of original problem.) It is easy to check that the weighted Chebyshev norm problem maintains the convexity if the original problems are convex; that is, all $f_{m}(\mathbf{x})$ and $h_{l}(\mathbf{x})$ are convex. Definitely, as an objective function has higher weight, it is liable to be emphasized relatively more than others.

We can envisage the linear combination of objective functions, such as $\delta_{1} f_{1}+\delta_{1} f_{1}$, in order to scalarize the multiobjective optimization problem. However the Chebyshev normalization is preferred to the linear combination due to the following reasons.

(1) The unit and scale of each objective are different: one expresses the number of handoffs and the other one expresses fairness value in terms of the number of spectrum. So we need to normalize the scales of the objectives.

(2) The linear combination does not guarantee the balance (fairness) between the two objectives. Surely, adjusting the weight of each objective may yield the balanced outcome but it is quite difficult to find the adequate weights. However, our approach yields balanced output without the weight values (i.e., delta_1 and delta_2). Then we can control the weight of each objective by adjusting the weight values. Surely, this approach is more straightforward.

If we denote $C v\left(f_{1}^{*}\right)$ and $C v\left(f_{2}\right)$ by $g_{1}$ and $g_{2}$, respectively, the problem $\Omega\left(f_{1}, f_{2}\right)$ is converted into a single objective optimization problem by Chebyshev norm problem.
Objectives. Consider the following:

Minimize $z$,

s.t.

$$
\begin{aligned}
& \left.C v\left(c_{1}\right): \ln \left(\begin{array}{c}
\sum_{j \in V \backslash\{i\}}\left(e^{\left(\beta_{i s}+\beta_{j s}+\gamma_{j s}-\gamma_{i s}\right)} G_{j k}^{s}+e^{\left(\beta_{i s}-\gamma_{i s}\right)} \sigma^{2}\right) \\
\left(G_{i k}^{s}\right)^{-1}\left(\operatorname{INSR}_{i}^{\max }\right)^{-1}
\end{array}\right)\right) \\
& \leq 0
\end{aligned}
$$

$\forall i \in V, \quad s \in S$

$$
\begin{gathered}
C v\left(c_{2}\right): \ln \left(\sum_{s \in S} e^{\left(\beta_{i s}+\gamma_{i s}\right)}\left(P_{i}^{\max }\right)^{-1}\right) \leq 0 \quad \forall i \in V, \\
c_{4}: \delta_{1} \times\left(\frac{-\sum_{i \in V} w_{i} \sum_{s \in S} \alpha_{i s}\left(\beta_{i s}-\ln \alpha_{i s}\right)-g_{1}^{*}}{g_{1 w}-g_{1}^{*}}\right) \leq z, \\
c_{5}: \delta_{2} \times\left(\frac{\ln \left(\sum_{s \in S} \sum_{i \in V} L_{i s} e^{-\beta_{i s}}\right)-g_{2}^{*}}{g_{2 w}-g_{2}^{*}}\right) \leq z,
\end{gathered}
$$

where $g_{1}^{*}$ is the optimal value when only $C v\left(f_{1}^{*}\right)$ is minimized and $g_{2}^{*}$ is the optimal value when only $C v\left(f_{2}\right)$ is minimized. Similarly, $g_{1 w}$ is the value of $C v\left(f_{1}^{*}\right)$ when only $C v\left(f_{2}\right)$ is minimized and $g_{2 w}$ is the value of $C v\left(f_{2}\right)$ when only $C v\left(f_{1}^{*}\right)$ is minimized. We apply $\alpha_{i s}$ obtained when solving $\Omega\left(f_{1}\right)$ by condensation. Therefore, prior to solving $\Omega\left(f_{1}, f_{2}\right)$, we should solve $\Omega\left(f_{1}\right)$ and $\Omega\left(f_{2}\right)$, respectively; the global solution of all these problems can be computed using a general NLP solving method such as interior point method [36].

4.3. Rounding Algorithm. Regardless of convexity and linearity, a problem with integer constraints is in general very hard to solve. In this paper, we apply a simple rounding method introduced in [34] for finding approximated integer solutions of $\Omega\left(f_{1}, f_{2}\right)$. We also use the rounding method to find the integer solutions of $\Omega\left(f_{1}\right)$ and $\Omega\left(f_{2}\right)$ for the purpose of evaluation. The algorithm of the rounding method is given as Algorithm 2.

In Step 2, we first solve NLP relaxed and convexified problems. In Step 3, rounding is performed with rounding distance $\xi$, which is the distance between $x_{i s}$ and its nearest integer. Then the feasibility is checked with the rounded solutions in Step 3. If the rounded solutions are not feasible, we reduce the rounding distance by $\xi$ (i.e., the rounding condition is made stricter) and perform the rounding and feasibility check again. Otherwise, in Step 5, we compute integer solutions using the original objective functions for the problem $\Omega\left(f_{1}\right)$ and $\Omega\left(f_{2}\right)$, and, for the problem $\Omega\left(f_{1}\right.$, $f_{2}$ ), we compute weighted summation of two normalized objective values since a single-valued metric is required for determining whether the iteration should proceed further or not. In Step 6, we update the best solution until the current iteration and fix the rounded solutions as parameters for the 
Step 1. best_sol:=0;

old_sol: $=0$;

$\beta:=\left\{\beta_{i s}: \forall i \in V\right.$ and $\left.\forall s \in S\right\}$;

$\gamma:=\left\{\gamma_{i s}: \forall i \in V\right.$ and $\left.\forall s \in S\right\}$;

Step 2. Solve NLP relaxed problem with the solution vectors $\beta$ and $\boldsymbol{\gamma}$;

Step 3. for each $\beta_{i s} \in \boldsymbol{\beta}$ begin

$$
\begin{aligned}
& \text { if }\left(\left|\exp \left[\beta_{i s}\right]-1.0\right| \leq \xi\right) \\
& x_{i s}:=1 ; \\
& \text { else } \\
& \quad x_{i s}:=0 ; \\
& \text { end }
\end{aligned}
$$

Step 4. Check the feasibility of the original problem with the rounded $\mathbf{x}$.

$$
\text { if not feasible begin }
$$$$
\xi:=\xi-\zeta
$$

end

$$
\text { goto Step 3; }
$$

Step 5. Compute integer solution int_sol of each objective function:

(1) For $\Omega\left(f_{1}\right)$,

$$
\text { int_sol: }=f_{1}=\sum_{i \in V} w_{i} \ln \left(\sum_{s \in S} x_{i s}\right) \text {; }
$$

(2) For $\Omega\left(f_{2}\right)$,

$$
\text { int_sol: }=f_{2}=\sum_{s \in S} \sum_{i \in V} L_{i s} x_{i s} \text {; }
$$

(3) For $\Omega\left(f_{1}, f_{2}\right)$,

$$
\text { int_sol: }=\delta_{1} f_{1 n}+\delta_{2} f_{2 n}
$$

where $f_{1 n}$ and $f_{2 n}$ are normalized $f_{1}$ and $f_{2}$ respectively computed by (16).

Step 6. if (int_sol > best_sol)

$$
\text { best_sol:=int_sol; }
$$

Step 7. for each $\beta_{i s} \in \boldsymbol{\beta}$ begin

$$
\text { if }\left(x_{i s}=1\right)
$$
End

$\boldsymbol{\beta}:=\boldsymbol{\beta}-\left\{\beta_{i s}\right\} ; / /$ if $x_{i s}=1$, fix $\beta_{i s}$ as a parameter for next rounding iteration.

Step 8. if $\left(\mid\right.$ old_sol $-i n t_{-}$sol $\left.\mid<\varepsilon\right)$

else

Terminate the algorithm;

old_sol: = int_sol;

goto Step 2;

Algorithm 2: Rounding algorithm.

next iteration in Step 7. Finally, if we conclude that the integer solutions are converged within a certain level, we terminate the rounding algorithm.

Although the rounding algorithm is quite efficient and easy to implement, it often produces infeasible solutions. To make matters even worse, it is extremely difficult to fit the rounded solutions into the constraints. However we observe that it works well for our problems since all the objective functions and constraints involve exponential function, which means that the NLP solver has a tendency to produce the variables bisected into very small or large values in order to seek the maximum (or minimum) results. To illustrate this phenomenon, we choose a small sensor topology where 5 sensors are distributed uniformly in a $20 \mathrm{~m} \times 20 \mathrm{~m}$ square and assume 20 idle spectrum units. We compute the NLP relaxed solutions of all the problems and plot them in Figure 1 where, for the problem $\Omega\left(f_{1}\right)$, the outputs of the condensation algorithm are plotted.
As shown in Figure 1, we observe that some variables are quite close to 1 and a few of them are exactly 1 , while some other variables are relatively very small. As a result, we can parameterize these bisected variables easily in the first rounding, which enables the next rounding to begin with reduced set of variables. In addition, we observe that the variables converge to feasible integer solutions within a few rounding iterations. We show this result in the next section.

\section{Numerical Experiments}

For the experiments, we consider a sensor field of $100 \mathrm{~m} \times$ $100 \mathrm{~m}$ rectangular area where 15 sensors are uniformly distributed and assume that all the sensors request transmissions. We generate each sensor's transmission target randomly, which may result in one-to-many or manyto-one communications as well as one-to-one. The other main experimental parameters are listed in Table 1 . We use 


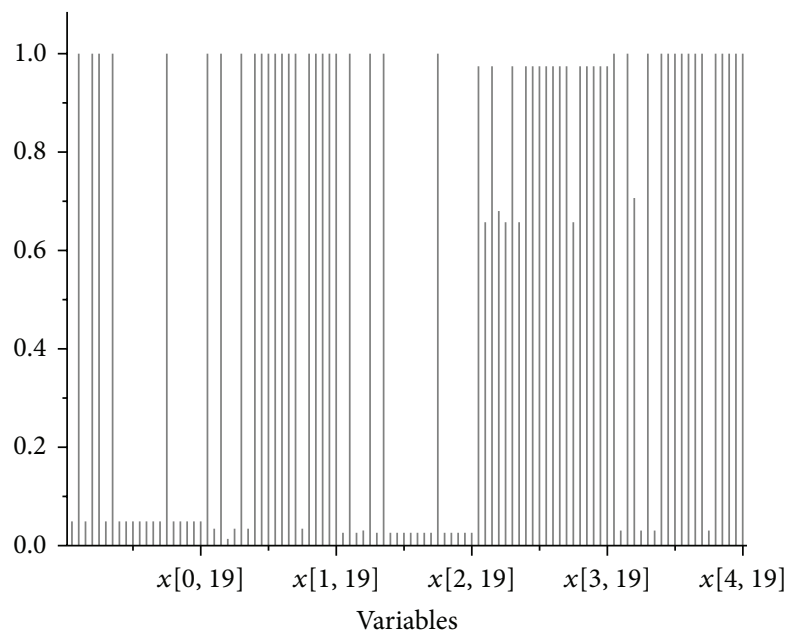

(a) For $\Omega\left(f_{1}\right)$

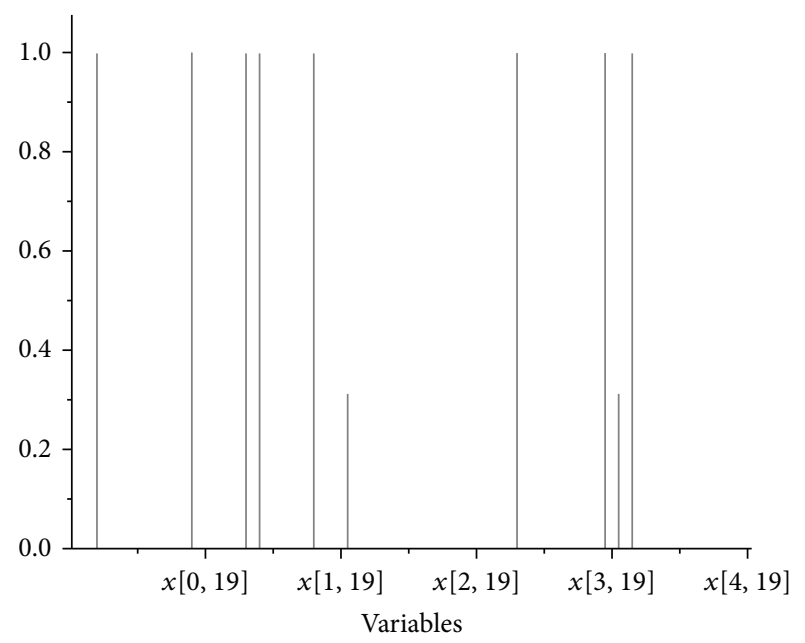

(b) For $\Omega\left(f_{2}\right)$

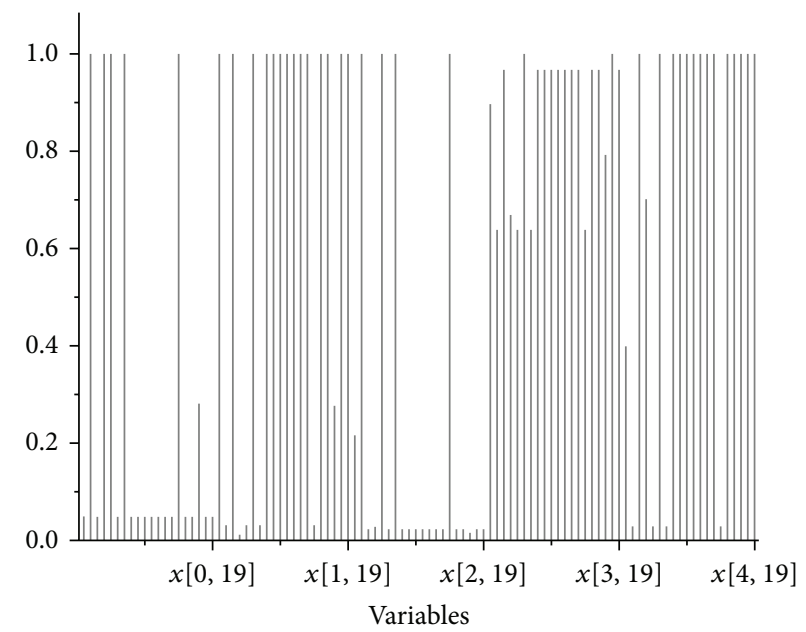

(c) For $\Omega\left(f_{1}, f_{2}\right)$

FIgURE 1: NLP relaxed solutions of variable $x_{i s}$ for all $i \in V$ and $s \in S$, for $\Omega\left(f_{1}\right)$. We plot the solution after the condensation.

the channel gain modeled as $G_{i j}=K_{0} \cdot 10^{\delta i j / 10} \cdot\left(d_{i j}\right)^{-v}$, where $\delta_{i j}$ is random Gaussian variables with zero mean and standard deviation equal to $6 \mathrm{~dB}, K_{0}=10^{3}$ that captures system and transmission effects such as antenna gain, and carrier frequency, and $d_{i j}$ is the distance between sensors $i$ and $j$, and $v$ is the power falloff factor. We let $v=3$. We implement all the experimental codes in $\mathrm{C}$ language using IPOPT [36] library that implements an NLP solver with interior point method. To illustrate that the condensation algorithm yields well converged NLP solution of the problem $\Omega\left(f_{1}\right)$ we measure the function value, $C v\left(f_{1}^{*}\right)$-defined in (14) - on each iteration of the condensation procedure, and Figure 2 shows the measured results. As shown in the graphs, we can observe that the function value $C v\left(f_{1}^{*}\right)$ converges to a stationary value as the condensation proceeds.

Table 2 lists the number of rounding iterations required to arrive at the given termination condition $\varepsilon=10^{-8}$. It is observed that the rounding algorithm converges to the termination condition within a few iterations as a rule except in the case of the problem $\Omega\left(f_{2}\right)$.

We evaluate the multiobjective solutions determined by the scalarization method by comparing with each single objective solution. Not only the integer solutions but also the NLP relaxed solutions are compared with varying the weight on each objective function, that is, $\delta_{1}$ and $\delta_{2}$. The results are plotted in Figure 3; we perform this evaluation with 60 different random channels that satisfy $95 \%$ maximum allowable error of $0.1 \mathrm{~dB}$ and plot the average values over those samples. The label of $X$-axis on each graph corresponds to the pair of weight on each objective function; from left to right, while $\delta_{1}$ increases, $\delta_{2}$ decreases. In the legend, "INTf1" and "INT-f2" are associated with the integer optimal values of the corresponding objective functions determined by the scalarization method and rounding algorithm; "NLPf1" and "NLP-f2" correspond to the NLP relaxed optimal values of the corresponding objective functions obtained after 
TABLE 1: Main experimental parameters.

\begin{tabular}{|c|c|}
\hline Experimental parameter & Value \\
\hline Number of sensors $(n)$ & 15 \\
\hline $\begin{array}{l}\text { Number of idle spectrum } \\
\text { units }\end{array}$ & 60 \\
\hline Weight $\left(w_{i}\right)$ & $\begin{array}{l}\text { Assign a uniform random } \\
\text { variable between } 1 \text { and } 20 \text { on } \\
\text { each sensor }\end{array}$ \\
\hline$L_{\text {hold }}$ & 0.1 \\
\hline$\sigma^{2}$ & $10^{-10} \mathrm{~W}$ \\
\hline$P_{i}^{\max }$ & $1 \mathrm{~mW}$ for all $i \in V$ \\
\hline $\mathrm{INSR}_{i}^{\max }$ & $-10 \mathrm{~dB}$ \\
\hline $\begin{array}{l}\text { Termination condition for the } \\
\text { condensation algorithm } \omega\end{array}$ & 0.1 \\
\hline Initial rounding distance $(\xi)$ & 0.9999 \\
\hline $\begin{array}{l}\text { Rounding distance decrement } \\
(\xi)\end{array}$ & 0.0001 \\
\hline $\begin{array}{l}\text { Termination condition for the } \\
\text { rounding algorithm }(\varepsilon)\end{array}$ & $\begin{array}{c}10^{-8} \text { for } \Omega\left(f_{1}\right) \text { and } \Omega\left(f_{1}, f_{2}\right) \text { and } \\
0 \text { for } \Omega\left(f_{2}\right)\end{array}$ \\
\hline \multicolumn{2}{|c|}{$\begin{array}{l}L_{\text {hold }} \text { : probability that each sensor holds a spectrum unit at its previou } \\
\text { transmission phase. }\end{array}$} \\
\hline \multicolumn{2}{|c|}{$\begin{array}{l}\text { TABLE 2: The number of rounding iterations required for the intege } \\
\text { solutions with the termination condition } \varepsilon \text {. }\end{array}$} \\
\hline Problem & $N=15$ \\
\hline$\Omega\left(f_{1}\right)$ & 2 \\
\hline$\Omega\left(f_{2}\right)$ & 9 \\
\hline$\Omega\left(f_{1}, f_{2}\right)\left(\delta_{1}=0.001, \delta_{2}=1.0\right)$ & 2 \\
\hline$\Omega\left(f_{1}, f_{2}\right)\left(\delta_{1}=0.01, \delta_{2}=1.0\right)$ & 2 \\
\hline$\Omega\left(f_{1}, f_{2}\right)\left(\delta_{1}=0.1, \delta_{2}=1.0\right)$ & 3 \\
\hline$\Omega\left(f_{1}, f_{2}\right)\left(\delta_{1}=1.0, \delta_{2}=1.0\right)$ & 3 \\
\hline$\Omega\left(f_{1}, f_{2}\right)\left(\delta_{1}=1.0, \delta_{2}=0.1\right)$ & 2 \\
\hline$\Omega\left(f_{1}, f_{2}\right)\left(\delta_{1}=1.0, \delta_{2}=0.01\right)$ & 2 \\
\hline$\Omega\left(f_{1}, f_{2}\right)\left(\delta_{1}=1.0, \delta_{2}=0.001\right)$ & 2 \\
\hline
\end{tabular}

the scalarization. In addition, the items with " $*$ " in the legend correspond to the single objective solutions. In this graph, we plot the NLP relaxed and integer solutions using the original objective functions, $f_{1}$ and $f_{2}$ in (2) and (3), respectively. The remarkable results are as follows: (i) both the NLP relaxed solutions and integer solutions of $f_{1}$ and $f_{2}$ in $\Omega\left(f_{1}, f_{2}\right)$ are apparently proportional to their respective weights; (ii) the solutions determined by the scalarization method are strictly bounded to each of the single objective solutions; (iii) the integer solutions are very close to the NLP relaxed solutions with the factor of less than 2 for both of the objective functions [14].

Next we measure how fairly the spectrum resources are allocated. To show this, we compute fairness index using (21) and plot it in Figure 4. Fairness index is widely used metric that measures the level of fairness. As it is close to 1 , the allocation is fairer. We also plot spectrum utilization using (22) in order to show how well the spectrum resources are

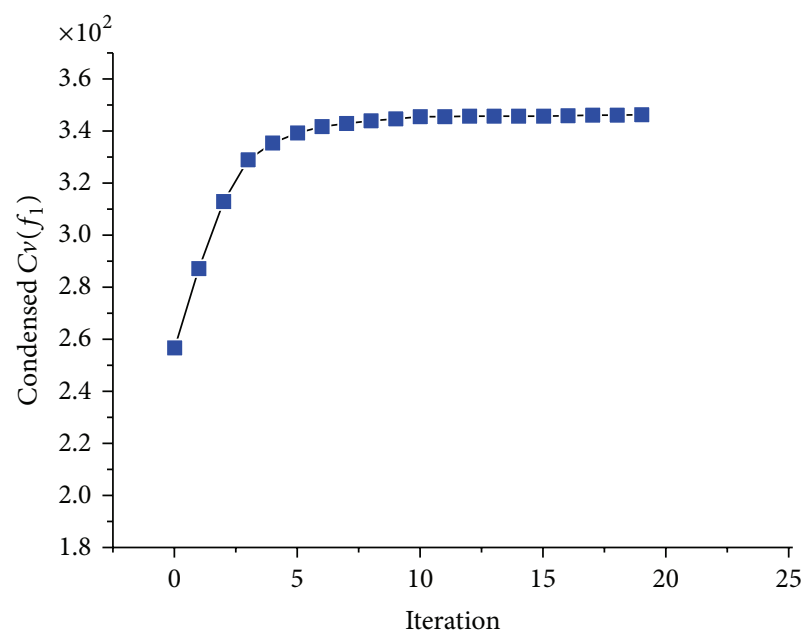

FIgure 2: The transition of the function value $C v\left(f_{1}^{*}\right)$ as the condensation proceeds.

utilized. We plot the average results obtained over 60 different random channels:

$$
\begin{gathered}
\mathrm{FI}=\frac{\left(\sum_{i \in V}\left(\left(\sum_{s \in S} x_{i s}\right) / w_{i}\right)\right)^{2}}{\left[n \sum_{i \in V}\left(\left(\sum_{s \in S} x_{i s}\right) / w_{i}\right)^{2}\right]} \\
\mathrm{SU}=\sum_{i \in V} \sum_{s \in S} \frac{x_{i s}}{|S|}
\end{gathered}
$$

where $n$ is the number of sensors.

Figure 4 shows that, as the weight on $f_{1}$ increases, also the fairness index increases generally. It is observed that the highest fairness index is yielded on $\langle 0.5,0.5\rangle$, and as $f_{1}$ gets more weight, it decreases slightly, which conforms to the attribute of the proportional fairness; if there are unallocated resources, maximal proportional fairness is achieved as allocating them despite of suffering the deterioration in the fairness index. On the other hand, maximal maxmin (or min-max) or strict fairness does not endure such deterioration.

To supplement with the evaluation results, we measure both integer and NLP relaxed function values of $f_{1}, f_{1}^{*}, f_{2}$, and $f_{2}^{*}$ and fairness index and spectrum utilization under different SINR constraints (i.e., with varying INSR ${ }^{\max }$ in constraint $c_{1}$ ) fixing the weight pair as $\langle 0.5,0.5\rangle$. We also measure the average value over 60 sampled random channels in these experiments. The results are plotted in Figure 5. As expected, it is noticed that all the function values and spectrum utilization increase as $\mathrm{INSR}^{\max }$ increases. With regard to the fairness, the highest fairness index is yielded when INSR $^{\max }=-10 \mathrm{~dB}$, and it is measured as 0.8563129 ; when $\mathrm{INSR}^{\max }=-7 \mathrm{~dB}$, the fairness index is measured as 0.8515264 that is lower than the case of INSR $^{\text {max }}=$ $-10 \mathrm{~dB}$. However, both the integer and NLP relaxed values of $f_{1}$ are higher when $\mathrm{INSR}^{\max }=-7 \mathrm{~dB}$ due to the higher spectrum utilization, which complies with the attribute of the proportional fairness, less fairness but higher utilization. 


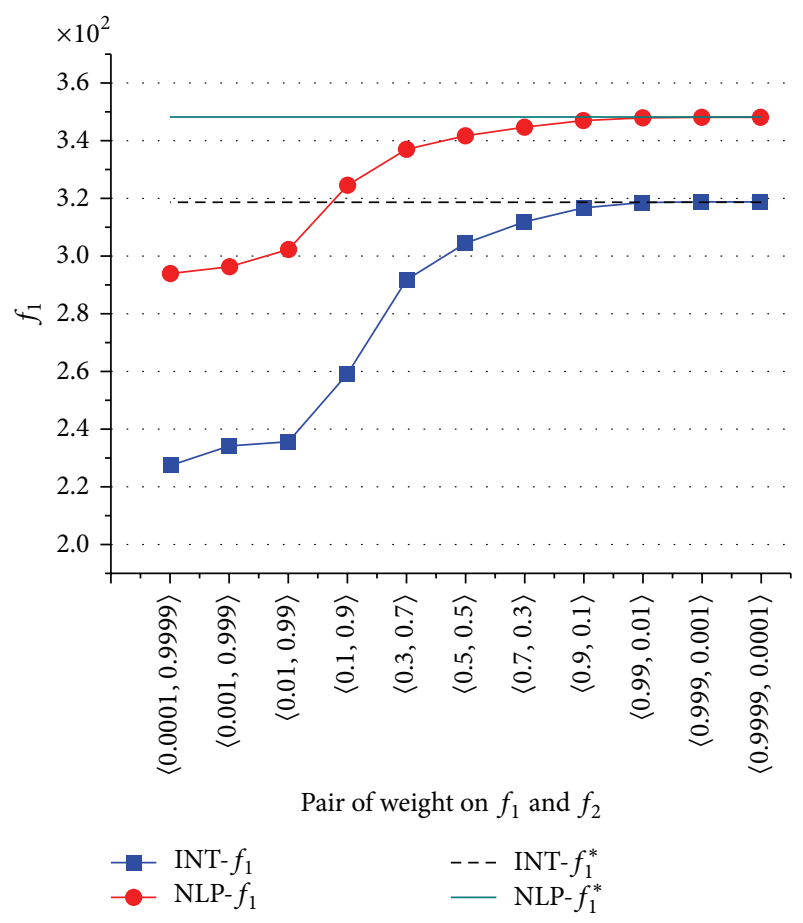

(a) Evaluation of $f_{1}$

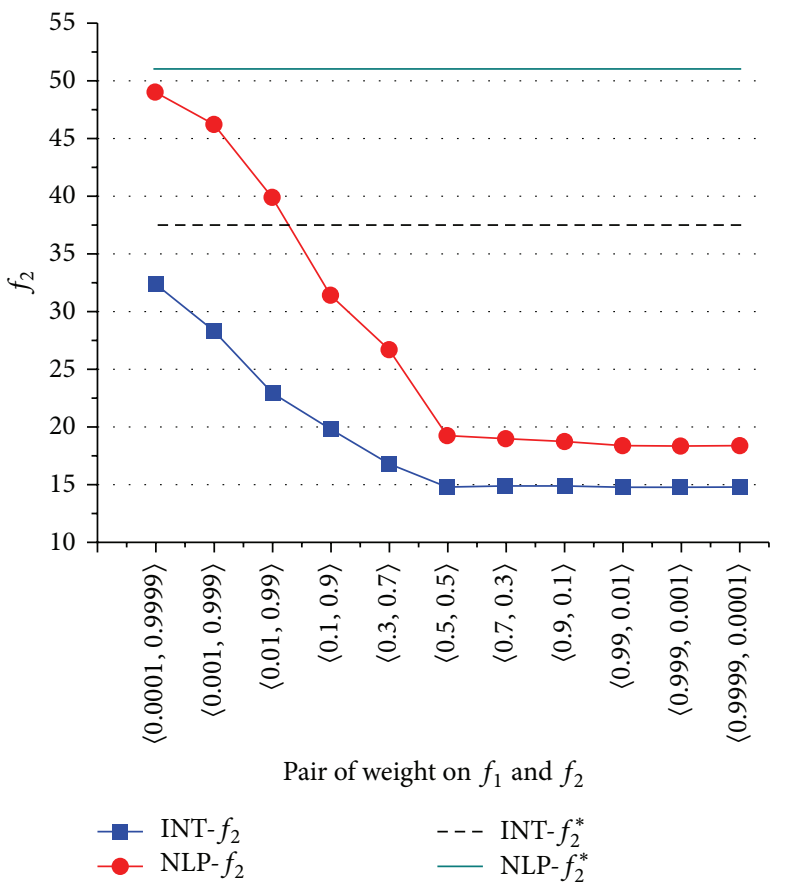

(b) Evaluation of $f_{2}$

FIGURE 3: Evaluation of $f_{1}$ and $f_{2}$ achieved by the scalarization method. The label of $X$-axis on each graph corresponds to the pair of weight on each objective function, $\left\langle\delta_{1}, \delta_{2}\right\rangle$. The integer solutions obtained by the rounding algorithm are also evaluated by comparing them to the NLP relaxed solutions.

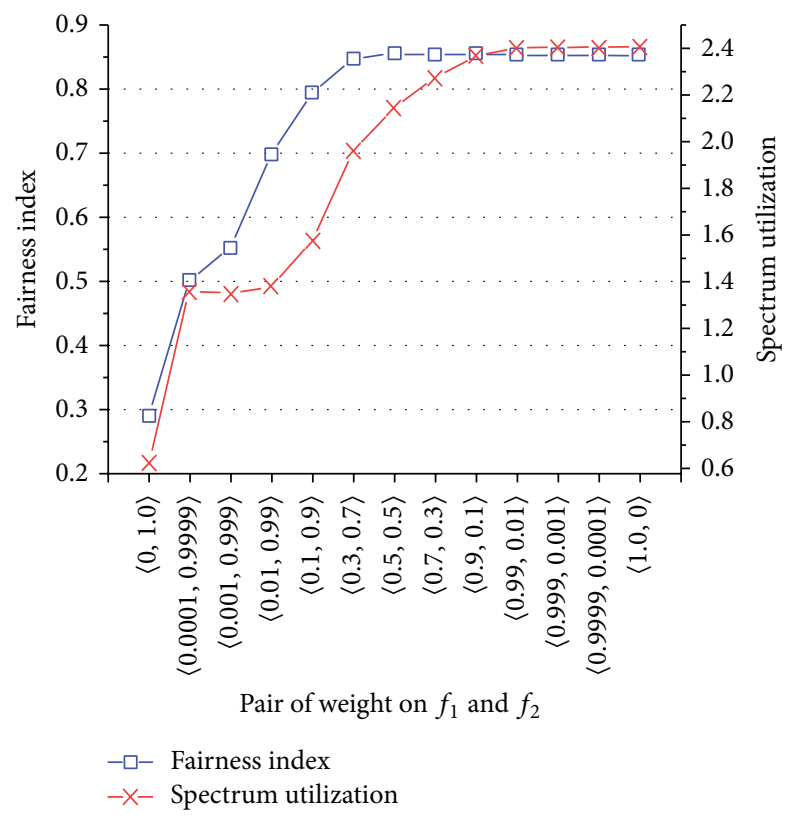

FIGURE 4: Fairness index and spectrum utilization according to the weights.

\section{Conclusions}

This paper deals with the problem of spectrum allocation in resource-constrained wireless sensor networks with the following goals: (1) maximizing fairness, (2) reflecting the priority among sensor data, and (3) avoiding unnecessary spectrum handoff. The first two goals are achieved by maximizing weighted proportional fairness. Therefore the problem has been formulated as an optimization with two different objective functions: multiobjective optimization. The multiobjective optimization is an indispensable tool for decision making if the benefit of a decision does not depend only on one object. The object is further mapped by one scalar-valued function, that is, scalarization. We deploy the scalarization method based on Chebyshev norm problem in order to maintain the convexity of the objective functions and constraints. Prior to applying the scalarization, the original objective functions are convexified by the arithmetic-geometric mean approximation and logarithmic change of decision variables. Also, all the constraints are transformed into log-sum-exp function form that is strictly convex. Furthermore, in order to find the good approximate integer solutions, a simple rounding algorithm is used, which is quite efficient due to the exponential feature of the problem. The numerical experiments illustrate the efficiency of the proposed solutions including the condensation and rounding algorithm. Furthermore, by the discreet adjustment of the weight on each objective function, the proposed algorithm performs well in achieving the balanced multiobjective solutions. Finally, it is illustrated that, given the weight of each sensor, the algorithm allocates spectrum units fairly as well as yielding high spectrum utilizations when $f_{1}$ has relatively higher weight than $f_{2}$. 


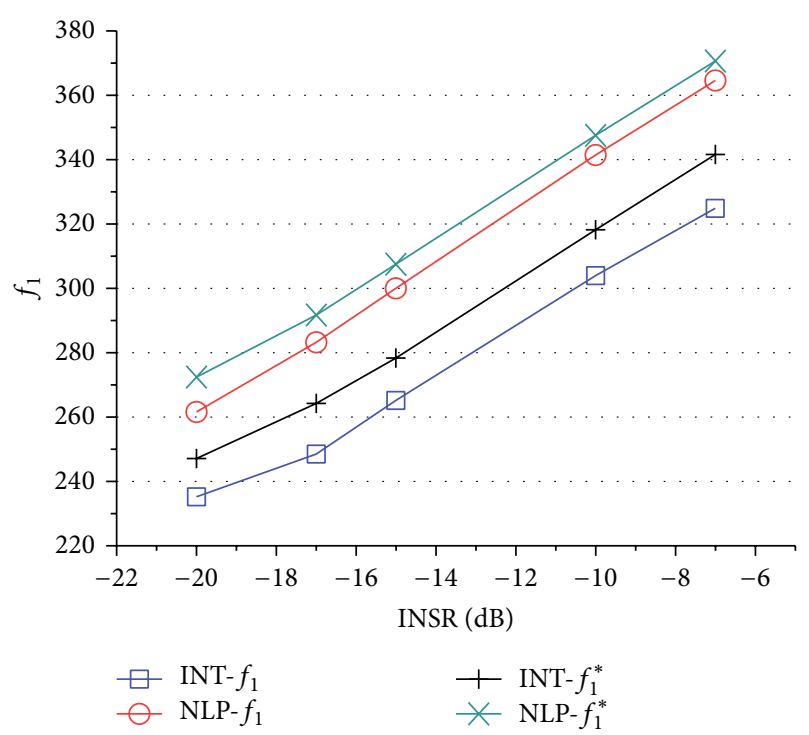

(a) Function value of $f_{1}$

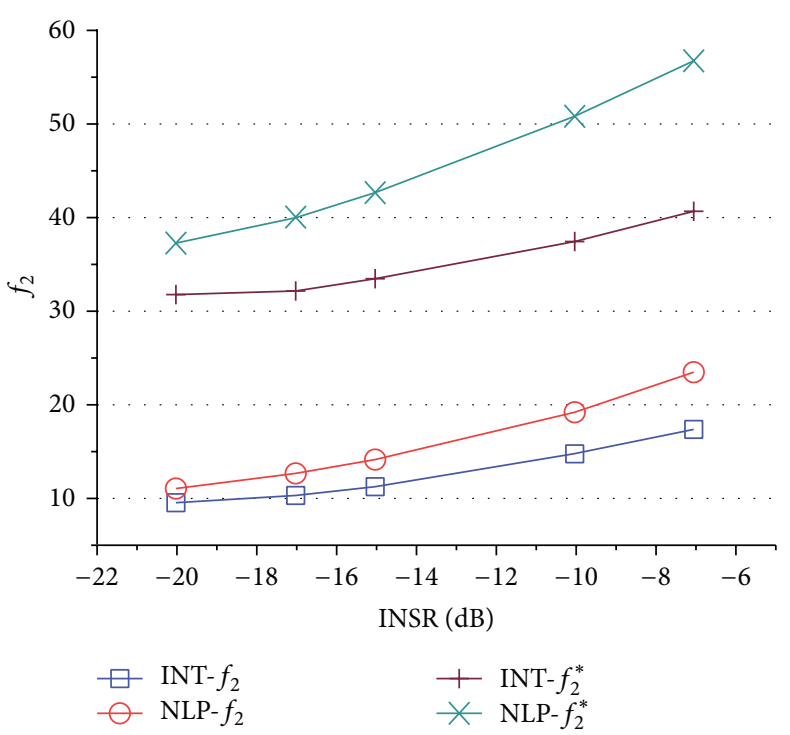

(b) Function value of $f_{2}$

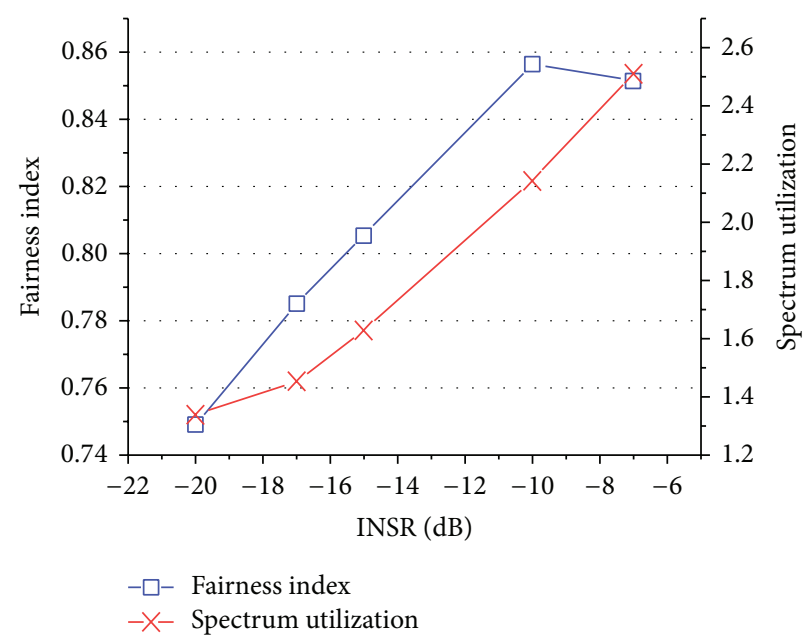

(c) Fairness index and spectrum utilization

FIgURE 5: Evaluation of function values of $f_{1}$ and $f_{2}$, fairness index, and spectrum utilization according to various INSR ${ }^{\max }$. The "INSR" on $X$-axis indicates INSR ${ }^{\max }$. We measure both integer and NLP relaxed function values of $f_{1}, f_{2}, f_{1}^{*}$, and $f_{2}^{*}$.

\section{Conflict of Interests}

The authors declare that there is no conflict of interests regarding the publication of this paper.

\section{Acknowledgment}

This research was supported by Basic Science Research Program through the National Research Foundation of Korea (NRF) funded by the Ministry of Education, Science and Technology (2012R1A1A4A01015777).

\section{References}

[1] Q. Zhao and B. M. Sadler, "A survey of dynamic spectrum access," IEEE Signal Processing Magazine, vol. 24, no. 3, pp. 7989, 2007.
[2] S. Haykin, "Cognitive radio: brain-empowered wireless communications," IEEE Journal on Selected Areas in Communications, vol. 23, no. 2, pp. 201-220, 2005.

[3] T. A. Weiss and F. K. Jondral, "Spectrum pooling: an innovative strategy for the enhancement of spectrum efficiency," IEEE Communications Magazine, vol. 42, no. 3, pp. S8-S14, 2004.

[4] H. Tang, "Some physical layer issues of wide-band cognitive radio systems," in Proceedings of the 1st IEEE International Symposium on New Frontiers in Dynamic Spectrum Access Networks (DySPAN '05), pp. 151-159, Baltimore, Md, USA, November 2005.

[5] C. Shahabi, "Janus-multi source event detection and collection system for effective surveillance of criminal activity," Journal of Information Processing Systems, vol. 10, no. 1, pp. 1-22, 2014.

[6] H. R. Lee, K. Y. Chung, and K. S. Jhang, "A study of wireless sensor network routing protocols for maintenance access hatch 
condition surveillance," Journal of Information Processing Systems, vol. 9, no. 2, pp. 237-246, 2013.

[7] K. Hwang and S. Nam, "Near real-time M2M communication for bidirectional AMR systems," Journal of Convergence, vol. 5, no. 2, 2014.

[8] I. F. Akyildiz, T. Melodia, and K. R. Chowdhury, "A survey on wireless multimedia sensor networks," Computer Networks, vol. 51, no. 4, pp. 921-960, 2007.

[9] M. Pioro and D. Medhi, Routing, Flow, and Capacity Design in Communication and Computer Networks, Morgan Kaufmann, Boston, Mass, USA, 2004.

[10] J.-Y. le Boudec, Rate Adaptation, Congestion Control and Fairness: A Tutorial, Ecole Polytechnique Federale de Lausanne (EPFL), 2008.

[11] E. Coersmeier, K. Hueske, M. Hoffmann, F. Leder, P. Martini, and H. Bothe, "Combining cognitive radio and software radio approach for low complexity receiver architecture," in Proceedings of the International Symposium on Advanced Radio Technologies, February 2007.

[12] I. F. Akyildiz, W. Lee, M. C. Vuran, and S. Mohanty, "NeXt generation/dynamic spectrum access/cognitive radio wireless networks: a survey," Computer Networks, vol. 50, no. 13, pp. 2127-2159, 2006.

[13] D. Lu, X. Huang, W. Zhang, and J. Fan, "Interference-aware spectrum handover for cognitiveradio networks," Wireless Communications and Mobile Computing, vol. 14, no. 11, pp. 1099-1112, 2014.

[14] H. Shokri-Ghadikolaei, F. Sheikholeslami, and M. NasiriKenari, "Distributed multiuser sequential channel sensing schemes in multichannel cognitive radio networks," IEEE Transactions on Wireless Communications, vol. 12, no. 5, pp. 2055-2067, 2013.

[15] I. Katzela and M. Naghshineh, "Channel assignment schemes for cellular mobile telecommunication systems: a comprehensive survey," IEEE Personal Communications, vol. 3, no. 3, pp. 10-31, 1996.

[16] G. Eichfelder, Adaptive Scalarization Methods in Multiobjective Optimization, Springer, Berlin, Germany, 2008.

[17] B. R. Marks and G. P. Wright, "A general inner approximation algorithm for nonconvex mathematical programs," Operations Research, vol. 26, no. 4, pp. 681-683, 1978.

[18] S. Gao, L. Qian, D. R. Vaman, and Q. Qu, "Energy efficient adaptive modulation in wireless cognitive radio sensor networks," in Proceedings of the IEEE International Conference on Communications (ICC '07), pp. 3980-3986, June 2007.

[19] S. Geirhofer, L. Tong, and B. M. Sadler, "A measurement-based model for dynamic spectrum access in WLAN channels," in Proceedings of the IEEE Military Communications Conference (MILCOM '06), pp. 1-7, IEEE, Washington, DC, USA, October 2006.

[20] Z. Han, Z. Ji, and K. J. R. Liu, "Fair multiuser channel allocation for OFDMA networks using Nash bargaining solutions and coalitions," IEEE Transactions on Communications, vol. 53, no. 8, pp. 1366-1376, 2005.

[21] D. Thilakawardana, K. Moessner, and R. Tafazolli, "Darwinian approach for dynamic spectrum allocation in next generation systems," IET Communications, vol. 2, no. 6, pp. 827-836, 2008.

[22] L. Cao and H. Zheng, "Stable and efficient spectrum access in next generation dynamic spectrum networks," in Proceeding of the 27th IEEE Communications Society Conference on Computer Communications (INFOCOM '08), Phoenix, Ariz, USA, April 2008.
[23] A. Konak, D. W. Coit, and A. E. Smith, "Multi-objective optimization using genetic algorithms: a tutorial," Reliability Engineering and System Safety, vol. 91, no. 9, pp. 992-1007, 2006.

[24] Y. Chen and J. Chen, "A fast subcarrier, bit, and power allocation algorithm for multiuser OFDM-based systems," IEEE Transactions on Vehicular Technology, vol. 57, no. 2, pp. 873-881, 2008.

[25] A. T. Hoang and Y. Liang, "Downlink channel assignment and power control for cognitive radio networks," IEEE Transactions on Wireless Communications, vol. 7, no. 8, pp. 3106-3117, 2008.

[26] J. Tang, S. Misra, and G. Xue, "Joint spectrum allocation and scheduling for fair spectrum sharing in cognitive radio wireless networks," Computer Networks, vol. 52, no. 11, pp. 2148-2158, 2008.

[27] L. Le and E. Hossain, "Resource allocation for spectrum underlay in cognitive radio networks," IEEE Transactions on Wireless Communications, vol. 7, no. 12, pp. 5306-5315, 2008.

[28] H. Boche and S. Stanczak, "Strict convexity of the feasible logSIR region," IEEE Transactions on Communications, vol. 56, no. 9, pp. 1511-1518, 2008.

[29] S. P. Algur and N. P. Kumar, "Novel user centric, game theory based bandwidth allocation mechanism in WiMAX," HumanCentric Computing and Information Sciences, vol. 3, article 20, 2013.

[30] U. Berthold and F. K. Jondral, "Guidelines for designing OFDM overlay systems," in Proceedings of the 1st IEEE International Symposium on New Frontiers in Dynamic Spectrum Access Networks (DySPAN '05), pp. 626-629, Baltimore, Md, USA, November 2005.

[31] M. Yoon, Y. Kim, and J. Chang, "An energy-efficient routing protocol using message success rate in wireless sensor networks," Journal of Convergence, vol. 4, no. 1, pp. 15-22, 2013.

[32] N. Ehsan and R. L. Cruz, "On the optimal SINR in random access networks with spatial reuse," in Proceedings of the 40th Annual Conference on Information Sciences and Systems (CISS '06), pp. 938-944, Princeton, NJ, USA, March 2006.

[33] S. Boyd and L. Vandenberghe, Convex Optimization, Cambridge University Press, Cambridge, UK, 2004.

[34] S. Boyd, S. Kim, and L. a. . Vandenberghe, "A tutorial on geometric programming," Optimization and Engineering, vol. 8, no. 1, pp. 67-127, 2007.

[35] S. S. Rao and T. I. Freiheit, "Modified game theory approach to multiobjective optimization," Journal of Mechanisms, Transmissions, and Automation in Design, vol. 113, no. 3, pp. 286-291, 1991.

[36] A. Wächter and L. T. Biegler, "On the implementation of an interior-point filter line-search algorithm for large-scale nonlinear programming," Mathematical Programming, vol. 106, no. 1, pp. 25-57, 2006. 

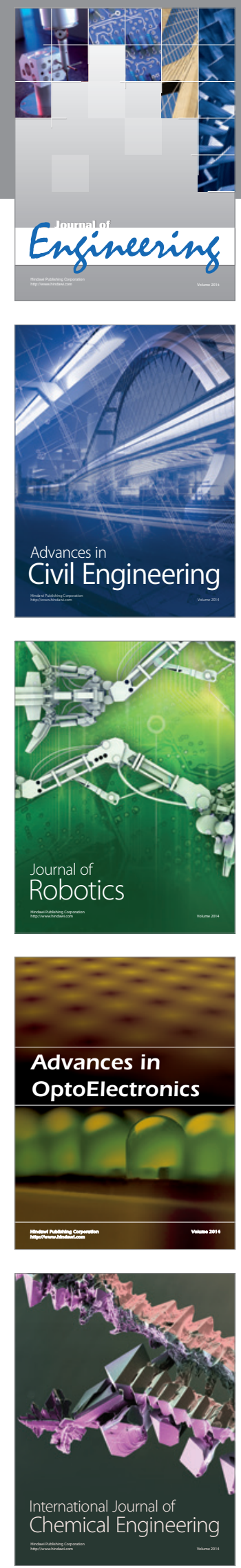

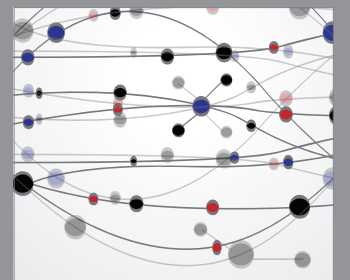

The Scientific World Journal
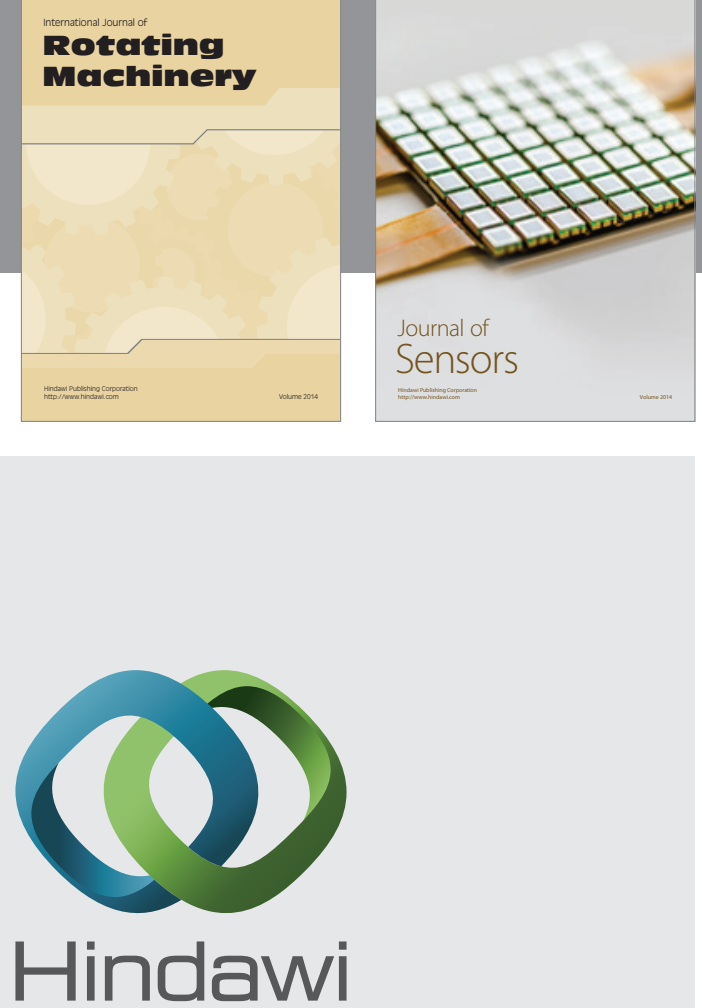

Submit your manuscripts at http://www.hindawi.com
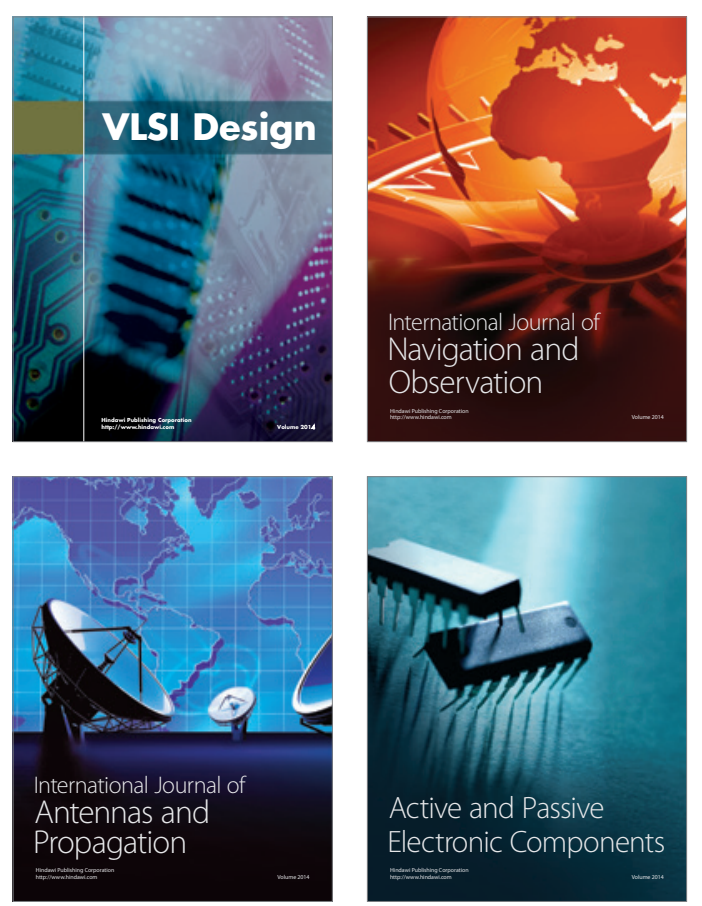
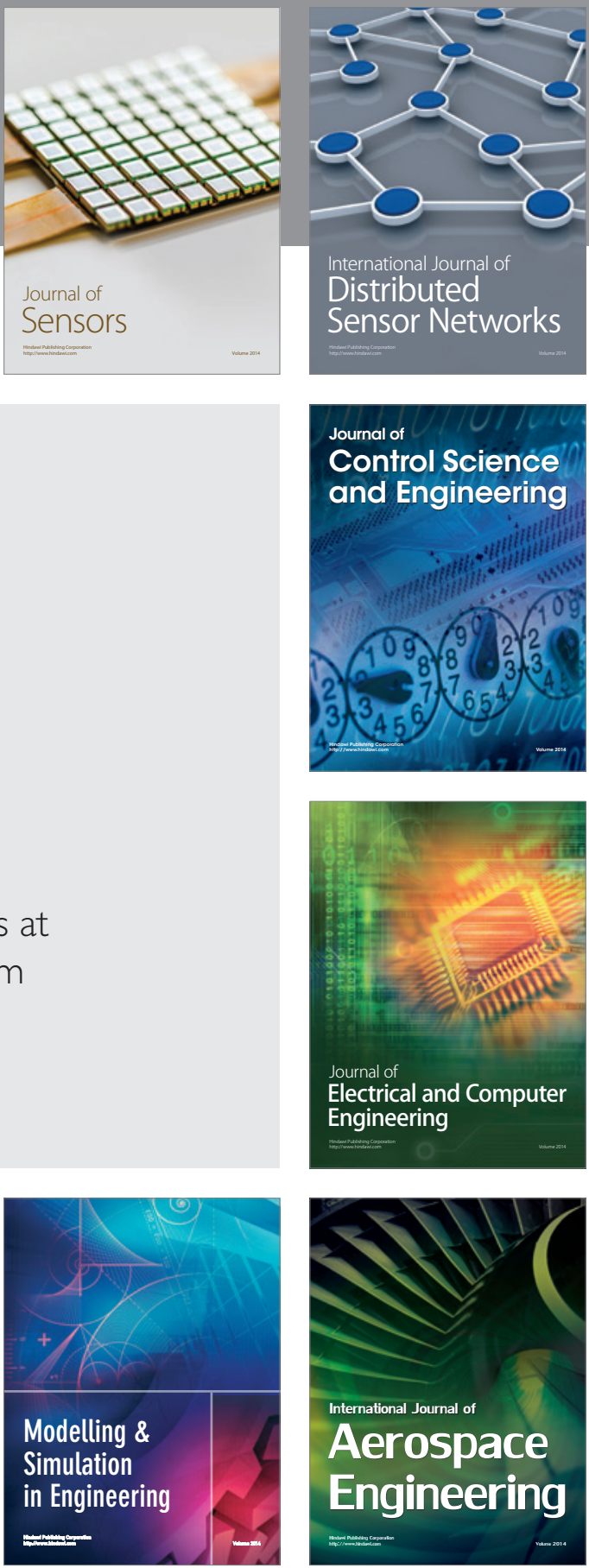

Journal of

Control Science

and Engineering
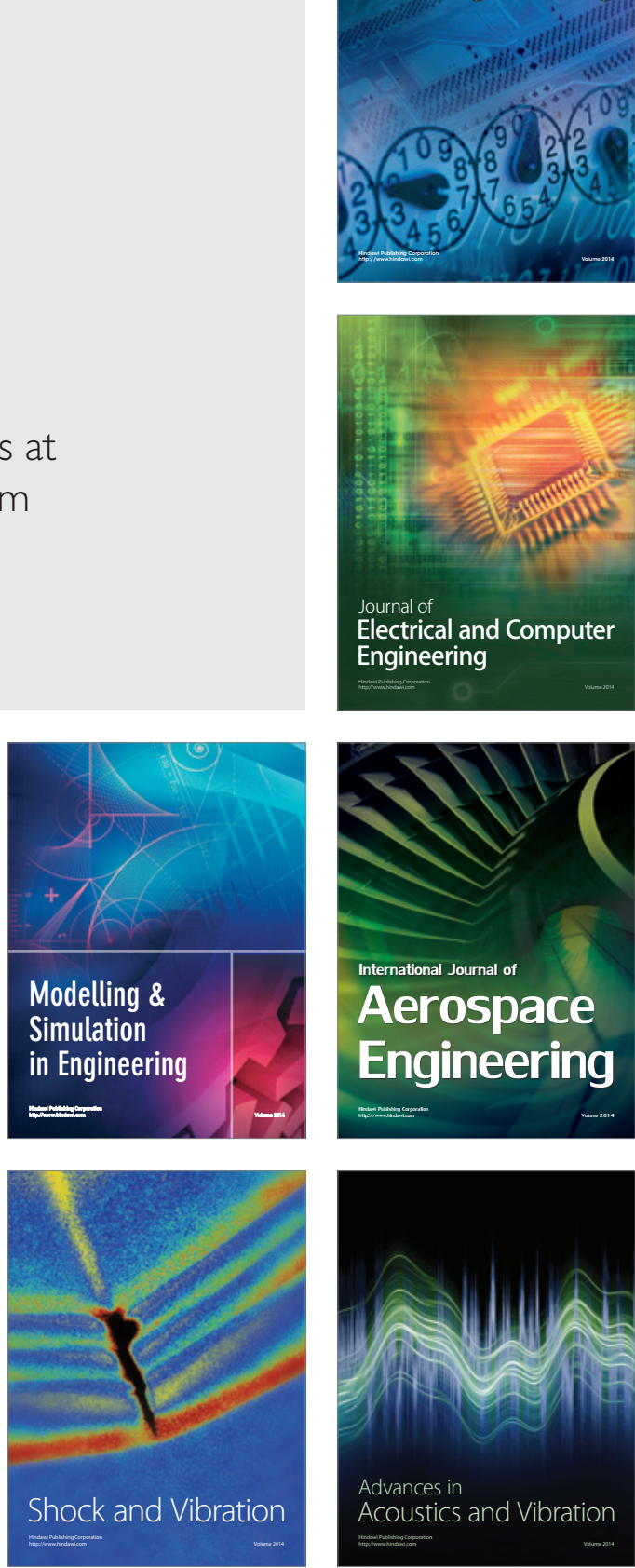